\title{
Telemedicine for gastrointestinal endoscopy: The Endoscopic Club E-conference in the Asia Pacific Region
}

\section{(1) $\circledast$}

\section{Authors}

Shiaw-Hooi Ho ${ }^{1}$, Rungsun Rerknimitr ${ }^{2}$, Kuriko Kudo ${ }^{3}$, Shunta Tomimatsu ${ }^{3}$, Mohamad Zahir Ahmad ${ }^{4}$, Akira Aso ${ }^{5}$, Dong Wan Seo ${ }^{6}$, Khean-Lee Goh ${ }^{1}$, Shuji Shimizu ${ }^{7}$

Institutions

1 University of Malaya Medical Centre - Department of Medicine, Kuala Lumpur, Malaysia

2 Chulalonkorn University - Medicine, Bangkok, Thailand

3 Kyushu University Hospital - Telemedicine Development Center, Fukuoka, Japan

4 University of Malaya Medical Centre - Department of Information Technology

5 Kyushu University - Medicine and Bioregulatory Science, Fukuoka, Japan

6 Asan Medical Center, University of Ulsan College of Medicine, Department of Internal Medicine, Seoul, Korea

7 Kyushu University Hospital - Department of Endoscopic Diagnostics and Therapeutics, Fukuoka, Japan

submitted 28.10.2016

accepted after revision 17.1.2017

Bibliography

DOI http://dx.doi.org/10.1055/s-0043-102935 |

Endoscopy International Open 2017; 05: E244-E252

(c) Georg Thieme Verlag KG Stuttgart · New York

ISSN 2364-3722

Corresponding author

Shiaw-Hooi Ho, University of Malaya Medical Centre, Department of Medicine, Faculty of Medicine, Kuala Lumpur Wilayah Persekutuan, Malaysia

shho@um.edu.my

\section{ABSTRACT}

Background and study aims An Endoscopic Club E-conference (ECE) was set up in May 2014 to cater to increased demand for gastrointestinal endoscopy-related teleconferences in the Asia-Pacific region which were traditionally organized by the medical working group (MWG) of Asia-Pacific Advanced Network. This study describes how the ECE meeting was run, examines the group dynamics, outlines feedback and analyzes factors affecting the enthusiasm of participants. It is hoped that the findings here can serve as guidance for future development of other teleconference groups.

Methods The preparation, running of and feedback on the ECE teleconference were evaluated and described. The country's economic situation, time zone differences, connectivity with a research and education network (REN) and engineering cooperation of each member were recorded and analyzed with regard to their association with participant enthusiasm, which was taken as participation in at least $50 \%$ of the meetings since joining. Associations were calculated using 2-way table with chi-square test to generate odds ratio and $P$ value.

Results To date, ECE members have increased from 7 to 29 (increment of $314 \%$ ). Feedback received indicated a high level of satisfaction with program content, audiovisual transmission and ease of technical preparation. Upper gastrointestinal luminal endoscopyrelated topics were the most favored program content. Those topics were presented mainly via case studies with a focus on management challenges. Time zone differences of more than 6 hours and poor engineering cooperation were independently associated with inactive participation ( $P$ values of 0.04 and 0.001 respectively).

Conclusions Good program content and high-quality audiovisual transmission are keys to the success of an endoscopic medical teleconference. In our analysis, poor engineering cooperation and discordant time zones contributed to inactive participation while connectivity with REN and a country's economic situation were not significantly associated with participant enthusiasm.

\section{Introduction}

Unbelievably, football has also kick-started many a beautiful thing. What was left from the 2002 Korea-Japan World Cup Tournament was not merely the memory of sportsmanship and friendship but something more solid that will continue to foster friendship and collaboration among the regional nations. Permanent large underwater fiber-optic cables that were laid during the tournament enabled high-speed internet connection between and beyond these 2 countries [1]. Barely a year after the tournament, multinational medical teleconferences in the Asia-Pacific region were initiated. The establishment of research and education networks (REN) in each country and the subsequent development of a digital video transport system (DVTS) led to initiation and booming of medical teleconferences among the regional countries. In the early years, medical teleconferences, organized through the Telemedicine Development Center (TEMDEC) of Kyushu University, were mainly surgery-related [1]. Gastrointestinal endoscopy-related activities were not as common. However, in recent years, gastrointestinal endoscopy-related teleconferences have become one of the most popular activities [2].

Traditionally, gastrointestinal endoscopy-related teleconferences were held twice yearly in conjunction with the biannual Asia Pacific Advanced Network (APAN) medical working group meeting which was initiated more than 10 years ago. However, 
due to the recent increase in the number of participating centers and the demand for more of such activities, a special interest group called Endoscopic Club E-conference (ECE) was formed in May 2014 to handle the increased demand for such activities. The establishment of this special interest group allows for meetings to be arranged outside the framework of the biyearly APAN meeting. In so doing, more centers will have the opportunity to engage in active participation such as performing live endoscopy demonstrations over teleconference, making presentations or being involved as assigned discussants. These educational activities are not only beneficial to the young gastrointestinal fellows but also play a role in retraining of established gastrointestinal endoscopists. The role of training and retraining has long been highlighted as a crucial element in ensuring a sustained competency of gastrointestinal endoscopists [3].

In view of the increasing popularity of such teleconference activity, it is felt that a description of the running of this teleconference special interest group is necessary to summarize the few key points in managing a teleconference group and to provide a guide for future development of other teleconference special interest groups. Various factors contributing to the level of participant enthusiasm were also analyzed and discussed.

\section{Methods}

\section{Group dynamics: Running of ECE}

Event preparation

An event calendar is available at the beginning of each year in the form of an online Google spreadsheet to allow activity and logistic planning. Nearer the day of the meeting, an eventspecific group email was generated to facilitate communication among the active participants. Technical test sessions were arranged as early as 2 weeks prior to the event. Alternative participation methods such as watching online streaming were also available for the newcomers and also for those who were unable to meet the minimum requirement during technical test sessions. To stimulate discussion, the topics and titles of each presentation were made available to the discussants and the participants several days before the meeting.

\section{On event day}

A Vidyo ${ }^{\circledR}$ teleconference system is generally used in all the ECE meetings. The connection normally starts 1 to 2 hours prior to the actual teleconference so that the engineering team can resolve loose ends. The physical set-up of the meeting is relatively simple. Both audiovisual recording and output devices are used along with the computing device, with is linked with the teleconference. The display interface was dynamic enough to capture all the participating centers during discussion and easy enough to be switched to presentation mode during presentation. Each teleconference lasted around 90 minutes \pm 10 minutes. Time keeping was viewed with utmost importance to the success of this meeting. The number of presentations varied from 4 to 6 in total. Depending largely on the number of presenters, each presenter was generally given approximately 7 to 8 minutes each for his or her presentation followed by discussion which took about the same amount of time. Two discussants were generally assigned to each presentation. Following each presentation, the assigned discussants gave comments and engaged in a further question and answer session with the presenter. General participants also were allowed to take part in the discussion whenever time permitted. Microphones were to be muted whenever not in use to reduce noise and avoid unnecessary echoes that might disrupt the teleconference. In view of the importance of this basic housekeeping rule, participants were generally reminded of this etiquette before and during the meeting.

Network and application performance were closely monitored by the engineers. Alternative communication pathways such as Adobe Connect and Skype were established concurrent with the teleconference to enable communication among the engineers during the event. Documentation of the event in the form of photos and assessment of audiovisual quality was done by each of the participating centers.

\section{Post-event}

Following the event, each of the participating centers was asked to submit photos taken at their sites and to provide feedback. The photos were kept by TEMDEC for documentation and storage purposes and can be retrieved (password protected) from their official website by their members (http://www.temdec.med.kyushu-u.ac.jp/eng/index.php). As for the feedback, an online feedback form (as outlined in the appendix) was available to participants immediately after the event and they were required to fill in the form within a stipulated time.

\section{Factors influencing participant enthusiasm}

In the second part of the study, general characteristics and level of participant enthusiasm among ECE members are described. The findings were tabulated in SPSS version 21.0 and baseline characteristics were analyzed. Mean of variables were calculated and presented. Factors such as the country's economic situation, time zone differences, connectivity with REN and engineering cooperation were described and analyzed against the level of participant enthusiasm to assess their relationship. In this aspect, a 2-way table with chi-square test was used to generate risk and $P$ value. A 2 -tailed $P$ value of $<0.05$ was considered statistically significant.

An assessment of the economic situation in each member's country was obtained from World Economic Outlook 2015 published by International Monetary Fund [4] while information on REN connectivity was obtained from the TEMDEC database or from members themselves. Time zone difference was measured as the difference between the local time zone and the Japanese time zone (where the event was hosted) according to Greenwich Mean Time (GMT). Normally the ECE meeting commences between 14:00 and 17:00 at GMT + 9 to allow participation from certain European centers with time zone differences of up to 8 hours. Engineering cooperation was assessed by the TEMDEC engineering team who were familiar with the running of ECE technical test sessions. Three objective criteria used in the assessment included replying to an email in timely fashion, 


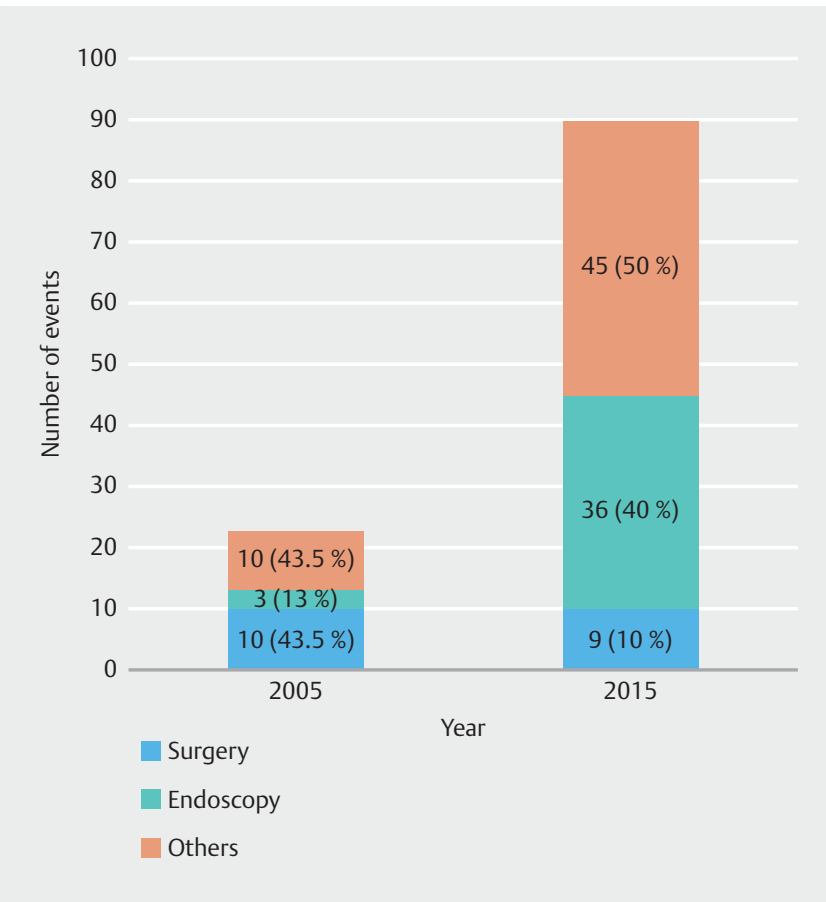

- Fig. 1 Stacked column bar chart depicting the growing number of teleconference activities and the increasing proportion of endoscopy-related events rrganized through TEMDEC

attending the technical test session and overall impression of the local engineers' skill. Each category was individually graded as satisfactory or non-satisfactory by 2 TEMDEC engineers who were blinded to each other's assessment. One mark was given for a satisfactory assessment and 0 mark for a non-satisfactory assessment. The maximum score was 6 and the minimum score was 0 . An overall satisfactory performance was given if the total assessment score was 4 and above whereas unsatisfactory performance if the total assessment score was 3 and below. As for participant enthusiasm, participation in greater than or equal to $50 \%$ of the activities since the date of joining was considered active and less than $50 \%$, inactive.

\section{Results}

\section{Group dynamics: Characteristics of and feedback from ECE Members}

Pooling information from the TEMDEC database over a span of 10 years, there were 10 surgery-related $(43.5 \%$ and 3 gastrointestinal endoscopy-related teleconferences (13.0\%) for 2005 and 9 (10.0\%) and 36 (40.0\%), respectively, for 2015 ( Fig.1). Hence, the last decade witnessed a surge in gastrointestinal endoscopy-related teleconferences in the region (1100\% increment).

Since the formation of this special interest group 2 years ago, the number of participating centers has grown from 7 to 29, an increment of $314 \%$ ( $\triangleright$ Fig. 2). Baseline characteristics of ECE members $(n=29)$ are shown in $>$ Table 1 . The majority of members are from Asian countries with Southeast Asian countries being the largest group (34.5\%) followed by East Asian

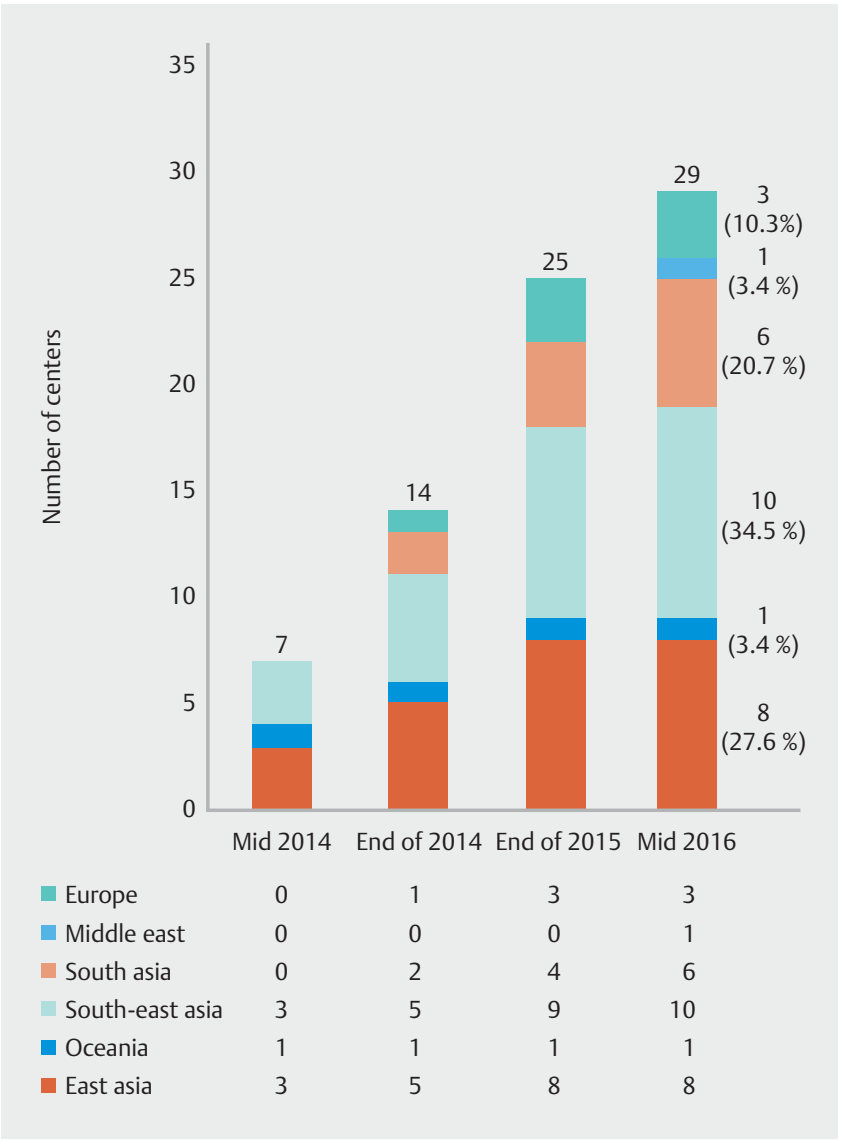

Fig. 2 Stacked column bar chart depicting expansion of ECE membership and region of member countries

(27.6\%) and South Asian countries (20.7\%). European members make up $10.3 \%$.

Feedback received from the 4 ECE meetings held in conjunction with the APAN Medical Working Group meeting, which occurred within 2 years after formation of ECE, indicated a high level of satisfaction in terms of audiovisual quality, ease of technical preparation and program content among participating members ( Fig.3). A grade of "very good" or "good" was seen in $98.9 \%$ in the aspect of image resolution, $94.7 \%$ in the aspect of image movement, $86.1 \%$ in the aspect of quality of sound and $100 \%$ in the aspect of program content. In the aspect of ease of technical preparation, $80.2 \%$ found it to be either "very easy" or "easy".

Overall, 10 ECE meetings were held with 51 presentations made. The program content was analyzed according to the subcategories of gastrointestinal endoscopy, point of interest in presentations and nature \& style of presentations ( $>$ Fig. 4 ). As can be seen in $>$ Fig. 4 a, upper gastrointestinal luminal endoscopy-related topics were the most popular $(n=18,35 \%)$ followed by endoscopic retrograde cholangiopancreatography [ERCP, $n=10(19 \%)$ ], lower gastrointestinal luminal endoscopy $(n=9,18 \%)$ and endoscopic ultrasound [EUS, $n=6(12 \%)$ ]. When analyzed according to the point of interest in presentations ( Fig.4b), most presentations were concerned with management challenges $(n=27,53 \%)$, followed equally by 
- Table 1 Background characteristics of ECE members.

\begin{tabular}{|l|l|c|}
\hline Characteristic & & Number (\%) \\
\hline Country's economic situation & Advanced & $14(44.3)$ \\
\hline & Developing & $15(55.7)$ \\
\hline Time zone difference with Japan at GMT +9 & Small ( $\leq 6$ hours $)$ & $27(93.1)$ \\
\hline & Huge (>6 hours) & $2(6.9)$ \\
\hline Connectivity with REN & Connected & $22(75.9)$ \\
\hline Engineering cooperation & Not connected & $7(24.1)$ \\
\hline Mean number of participating centers in each meeting & Satisfactory & $18(62.1)$ \\
\hline Mean number of meeting(s) taken part by each center & Unsatisfactory & $11(37.9)$ \\
\hline Mean level of participation since joining ECE $(\%)$ & & $11.2 \pm 2.7$ \\
\hline Participant enthusiasm & & $3.9 \pm 2.9$ \\
\hline
\end{tabular}

diagnostic challenges $(n=12,23.5 \%)$ and demonstration of new techniques or devices ( $n=12,23.5 \%$ ). In term of styles of presentation ( $\triangleright$ Fig. 4 c), most were case presentations ( $n=44$, $86 \%$ ) and some were didactic lectures ( $n=6,12 \%)$. A live demonstration took place only once $(2 \%)$ within this period of time.

\section{Factors influencing participant enthusiasm}

In terms of a country's economic situation, $44.3 \%$ of the members belonged to countries with an advanced economy and $55.7 \%$ had a developing economy. Most members (93.1\%) were from regions with a small time zone difference and only a small percentage of the members $(6.9 \%)$ were from a region with a time zone difference of more than 6 hours. The majority of the members $(75.9 \%)$ were linked with REN, and only about a quarter of ECE members (24.1\%) were members without REN connectivity. Engineering cooperation was found to be satisfactory in $62.1 \%$ of the members and unsatisfactory in the remaining $37.9 \%$ of the participants.

On average, 4 to 5 meetings were held each year. The mean number of participating centers in each ECE meeting was 11.2 \pm 2.7 . The mean number of meetings attended by each center was 3.9 \pm 2.9 . However, because not all of them joined ECE from the beginning, their level of participation was assessed individually from the time they joined. The level of participation was presented as number of attended meetings over the number of ECE activities held (in percentage) since joining. The mean level of participation by each center according to this calculation was $59.9 \pm 28.5 \%$. When active participation was taken as greater than or equal to $50 \%$ participation from the time of joining, about two-thirds of centers (65.5\%) were found to have active participation while the remaining one-third (34.5\%) had inactive participation.
When analyzed against participant enthusiasm, discordant time zones (i.e. more than 6 hours from where the event host is) and unsatisfactory engineering cooperation were independently found to be significantly associated with inactive participation with odds ratio (OR) of 3.38 [95\% confidence interval (CI) 1.89-6.04, $P=0.04]$ and $21.33(95 \% \mathrm{Cl} 2.95-154.55, P=$ 0.001 ) respectively ( $>$ Fig. 5 ). A country's economic situation or connectivity with REN was not found to be a significant association factor ( $P$ values of 0.09 and 0.15 , respectively).

\section{Discussion}

\section{Overview}

Teleconferences offer a unique learning experience whereby participants do not need to travel far to meet at a common venue, thus saving huge amounts of time and cost. A more interesting finding was reported by Holmner et al who showed that by replacing a physical meeting with telemedicine, carbon reduction can be achieved, thus offering a greener choice than conventional meeting [5]. As shown in the decade-long result, endoscopy-related teleconferences are gaining popularity [2]. Recent advances in the field of diagnostic and therapeutic endoscopy "prompted" a wave of mutual learning from each other [2,6-8]. Chung et al accurately pointed out that the conventional ways of disseminating knowledge via publication in journals and books cannot match the pace of rapid development in the field of therapeutic endoscopy and laparoscopic surgery [9]. Publishing or spreading of knowledge via electronic media (website, teleconference, etc.) seems to fill the gap. Furthermore, because of the unique quality of live imagery and various endoscopic skills that can be demonstrated in a rather simple manner and short duration of time, gastrointestinal endoscopy has become one of the favorite sessions during medical teleconferences. 
Q1. Image resolution

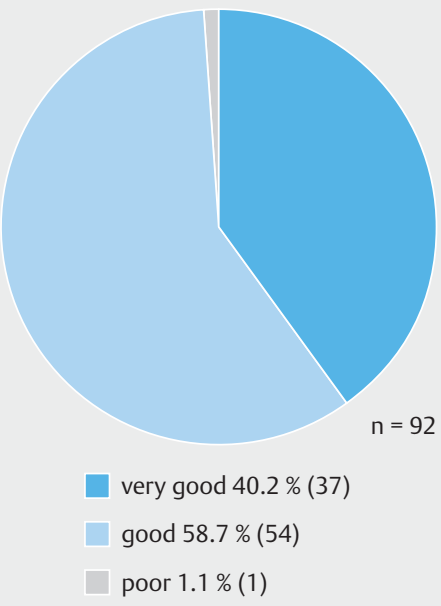

Q4. Program

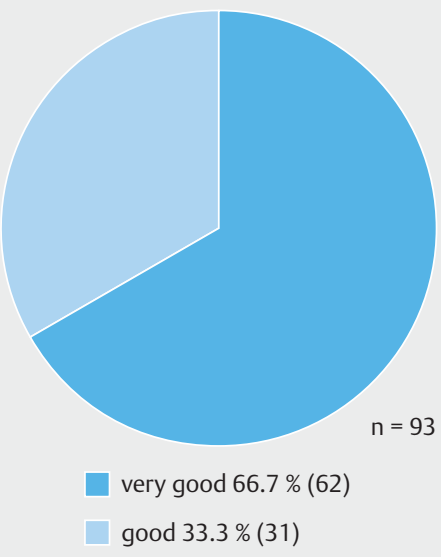

Q2. Image movement

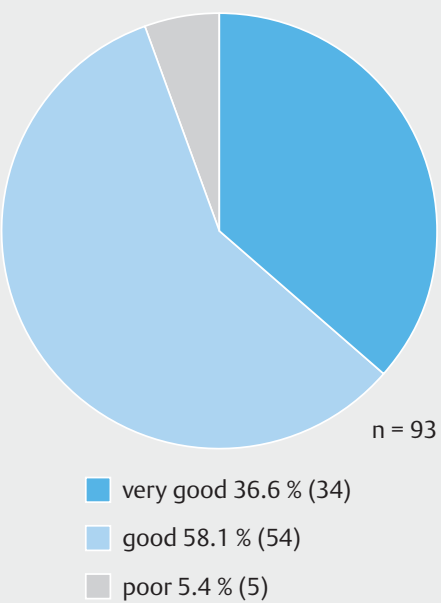

Q5. About the preparations

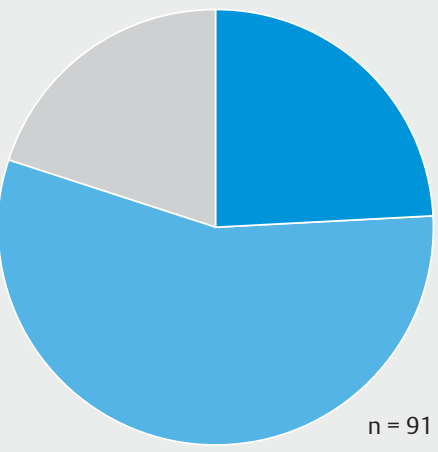

very easy $24.2 \%$ (22)

easy $56.0 \%(51)$

time consuming $19.8 \%$ (18)

- Fig. 3 Piecharts indicating Feedback Responses from 4 ECE meetings held in conjunction with APAN Medical Working Group meeting Number in parentheses "()" and " $n$ " refer to total number of respondents in the 4 ECE meetings

\section{Group dynamics: Running of ECE}

The overall high satisfactory performance in terms of audiovisual transmission owed its success to the support rendered by the ECE engineering team and TEMDEC. The meticulous test sessions organized prior to the event and the frequent communication between the engineers helped minimize misunderstanding and knowledge gaps. Furthermore, availability of an online event calendar allowed participants to plan their activity ahead and resolve logistical issues such as booking IT personnel, computing equipment and meeting hall. This will inevitably encourage more participation from the ECE members.

\section{Group dynamics: Characteristics of ECE members and feedback}

ECE members are mainly from Asian countries (89.7\%). This is more because of logistic reasons as TEMDEC is located in Kyushu, Japan. Through regional gastrointestinal endoscopyrelated conferences and workshops, mutual collaboration and friendships were formed in this region. Being invited to participate by someone in a collaborative circle is a strong "draw". Furthermore, the small time zone differences also encourage participation from centers around the Asian region.

From the feedback analysis, a high level of satisfaction with the program content was observed. That indirectly indicates that the participants found the program to be helpful in their clinical practice. It is believed that this aspect could be the single most important factor that contributed to the popularity of ECE. As mentioned earlier, the opportunity for retraining is equally important to established gastrointestinal endoscopists. 


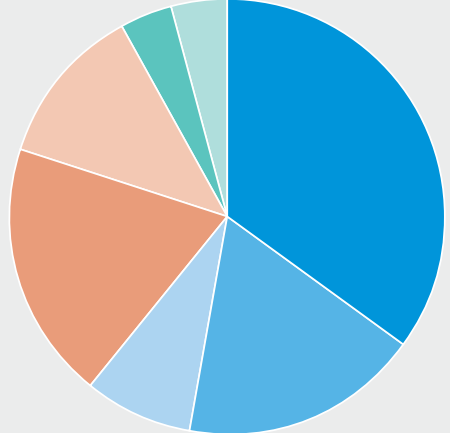

Upper GI luminal endoscopy 35 \% (18)

Lower Gl luminal endoscopy 18 \% (9)

Mixed upper \& lower luminal endoscopy 8 \% (4)

ERCP $19 \%(10)$

EUS $12 \%(6)$

Mixed ERCP \& EUS 4 \% (2)

Others $4 \%(2)$

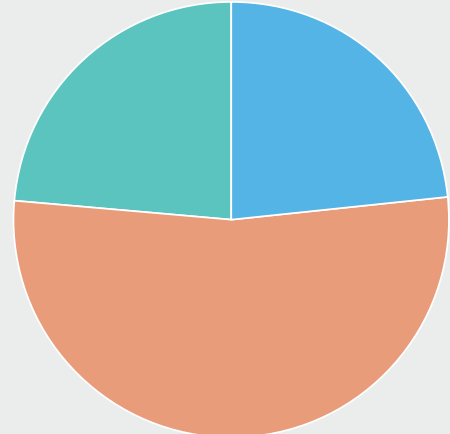

Diagnostic challenges $23.5 \%$

Management challenges $53 \%$

New techniques/devices $23.5 \%$

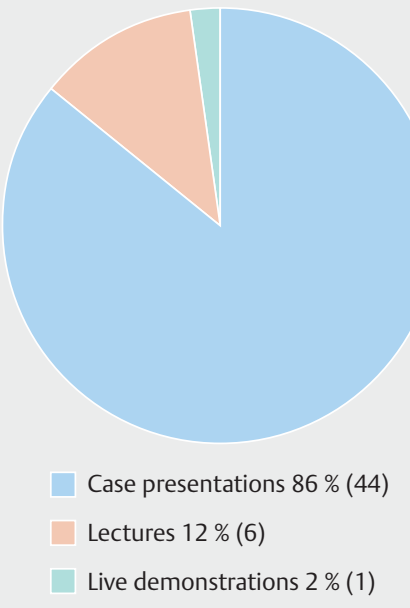

- Fig. 4 a Program content according to subcategories of gastrointestinal endoscopy. b Program content according to point of interest in presentations. c Program content according to nature and style of presentations.

As new technologies and techniques are introduced now and then, established endoscopists need to improve their capabilities [3]. Our program provides a platform for the established endoscopists to be exposed to the latest development in gastrointestinal endoscopy.

Upper gastrointestinal endoscopy-related topics seemed to garner more interest, perhaps due to the high burden of Helicobacter pylori gastritis, gastric cancer and other upper gastrointestinal diseases in the Asia Pacific region. Case presentations with focus on management challenges were the common style of presentation. These presentations based on real-life scenarios with management challenges stirred in-depth discussion among clinicians. To a lesser degree, presentations that focused on diagnostic challenges and demonstration of new techniques or devices were also not uncommon. Other styles of presentation included didactic lecture and live demonstration. Didactic lectures were usually delivered by the key-opinion-leader in a particular field as identified by the participants and those authorities, too, were believed to be a strong attraction factor. Due to time limitations and a requirement for more technical preparation, live demonstration was not as popular. However, with careful time management and breaks, live demonstrations can potentially be highly sought-after content in an ECE meeting. Recently, there is increasing concern that a conventional live demonstration may actually be risky to patients because the invited external faculty may be tired from long overseas trips and have to perform demonstrations in an unfamiliar environment, which inevitably increases their anxiety level. Furthermore, the experts frequently are distracted by questions [10]. Hence, broadcasting live demonstrations from a faculty member's own facility eliminates, in part, the un- favorable element of working in an unfamiliar environment and negates the need to acquire temporary medical licenses and special malpractice coverage. Because the benefit of such remote transmission is undisputable, it is no wonder that a recent survey among participants at a gastrointestinal endoscopy congress recorded a high majority with preference towards such a method of live demonstration (80.6\%) over the conventional style (3.3\%), whereby all the invited faculty performed endoscopy in a single hospital [11].

\section{Factors influencing participant enthusiasm}

In our study, a country's economic situation is taken as a surrogate marker of a country's general healthcare standard. Centers from countries with a developed economic platform are likely to have high healthcare standards and are deemed to be more advanced in terms of their healthcare practice. These advanced centers are more likely to be better off in terms of their technical know-how and they are also more likely to be in the forefront of research. Thus, other centers could benefit from knowledge shared by more advanced centers. Our study showed that centers from countries with developing economies had more active participation although it did not reach statistical significance. That observation can also be explained simply by the fact that more members are from Asian countries with developing economies.

Technologies used more frequently in the past included multiple long coaxial cables, fiber optic video-link cables, multiple integrated services digital network (ISDN) lines, microwave and satellite transmission [9]. DVTS and H.323 are the main technologies used in recent years to secure network-based connections $[1,2,12,13]$. In the era of DVTS a decade ago, 


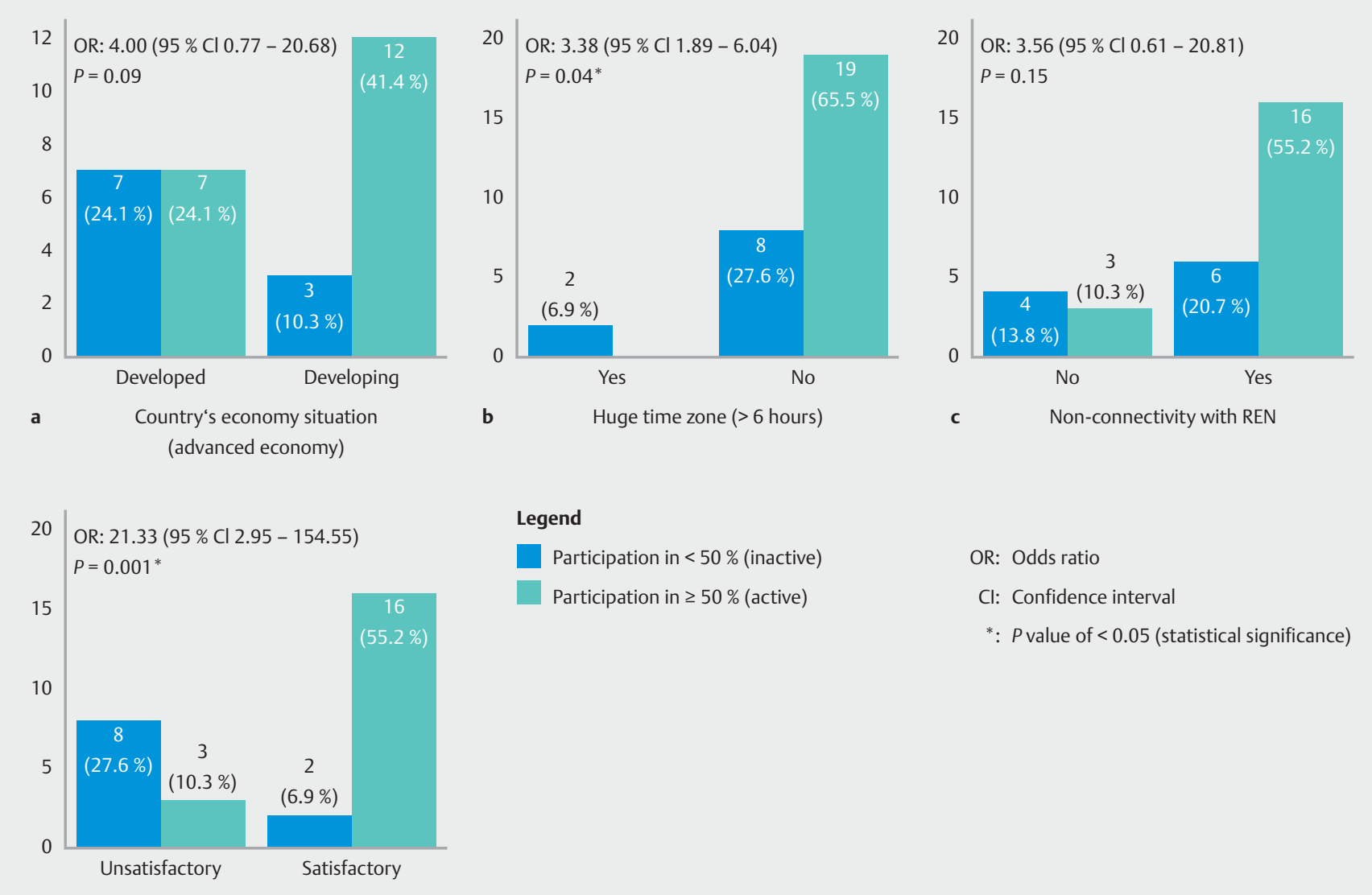

d Unsatisfactory engineering co-operation

- Fig. 5 Factors associated with inactive participation.

REN connectivity was an important prerequisite for a medical teleconference $[1,6,7,12]$. Without the large bandwidth provided by REN, it was impossible to achieve a connection via DVTS. Recently, taking advantage of the H.264 scalable video coding format, which resulted in adaptive bandwidth requirement, the Vidyo ${ }^{\circledR}$ teleconference system is gaining much popularity $[2,12,13]$. The advent of the Vidyo ${ }^{\circledR}$ teleconference system "revolutionized" current teleconference practice. Once an event is hosted by a center with Vidyo ${ }^{\circledR}$ infrastructure, all the invited participants can join via their personal computing devices using either REN or even a commercial network. With use of this system in ECE teleconferences, more and more medical centers without REN connectivity have been able to participate in teleconferences. In our study, approximately one-quarter of ECE members were without REN connection. Statistical analysis did not reveal any significant relationship between REN connectivity and higher participant enthusiasm. However, REN connection is still more preferred than a commercial network due to its large bandwidth and robust network stability.

Time zone differences remain one of the biggest challenges in organizing any teleconference. Because that factor is nonmodifiable, it requires mutual compromise and understanding from the participating centers. Not unexpectedly, centers with discordant time zones were found to be more inactive, perhaps due to the logistic disadvantages as they had to make more adjustments and compromises. However, careful and thoughtful adjustment of teleconference schedules could still draw participation from such "disadvantaged" centers.

Assessment of engineering cooperation included evaluation of timely response to email correspondence, attendance at technical test sessions and overall impression of an engineer's skill. It is interesting to note that unsatisfactory engineering cooperation was associated with lower participant enthusiasm. Engineers play an important role in establishing a favorable environment for a teleconference. When the environment is not conducive because of unsatisfactory audio and video quality, disrupted connection, etc., physicians become discouraged and may eventually lose interest completely in the endeavor. Of course, the cause and effect can be explained vice versa as well. Lack of interest from physicians may discourage engineers from taking a more active role in setting up a conducive teleconference environment. In a nutshell, the success of telemedicine depends on a synergistic effort from both engineers and physicians. Fortunately, engineering cooperation is a modifiable factor and can be improved through further communication among engineers and better understanding of the value of teleconference among physicians. 


\section{Limitation}

One of the limitations was the relatively short period of observation for newcomers who may show high enthusiasm only in the beginning. Of course, there are other factors that may affect participant enthusiasm such as the efficiency of the organization and running of ECE, continued support from TEMDEC, etc. which are beyond the scope of this study.

\section{Conclusions}

We have witnessed a rapid surge in the need for medical teleeducation among healthcare providers. Go et al predicted 20 years ago that medical teleconferencing of today may become as common in the workplace as copiers and fax machines [14]. Although we are still far from his vision, we are certainly steps closer to realization of this dream.

In conclusion, the popularity of a medical teleconference can be attributed to various factors. Program content is and will always be one of the most important factors that draw participation. In our experience, gastrointestinal endoscopy-related content was shown to be highly popular and sought after. Second, a high level of satisfaction with audiovisual transmission and ease of technical setup is in line with the maturing of the teleconference technology and the result of extensive technical test sessions by the engineering team. Both good scientific content and high-quality audiovisual transmission will continue to drive physicians to take part in medical teleconferences. From our analysis, poor engineering cooperation and discordant time zones discourage participation. It is hoped that the strong "draw" factors will overcome the non-modifiable "push" factor of discordant time zones and continue to attract participation from these "distant" centers. Compromise and adjustment will then be crucial in order to make medical teleconferences across large time zone differences a sustained academic endeavour.

\section{Acknowledgement}

This work was supported by JSPS Core-to-Core Program, B. Asia-Africa Science Platforms and KAKENHI Grant Number $15 \mathrm{~K} 08556$ and $16 \mathrm{H} 02773$. The corresponding author would like to acknowledge Malaysian Research and Education Network (MYREN) of the Malaysian Ministry of Higher Education and Telemedicine Development Center of Kyushu University for their supports in making this activity a success.
Competing interests

None

References

[1] Shimizu S, Nakashima N, Okamura K et al. International transmission of uncompressed endoscopic surgery images via superfast broadband Internet connections. Surgical endoscopy 2006; 20: 167 - 270

[2] Shimizu S, Kudo K, Antoku Y et al. Ten-year experience of remote medical education in Asia. Telemedi J E-health 2014; 20: 1021 -6

[3] Adler DG, Bakis G, Coyle WJ et al. Principles of training in gastrointestinal endoscopy. Gastrointest Endosc 2012; 75: 231 - 235

[4] Fund IM. World Economic Outlook: Uneven Growth - Short- and Long-Term Factor. Washington; 2015

[5] Holmner A, Ebi KL, Lazuardi L et al. Carbon footprint of telemedicine solutions-unexplored opportunity for reducing carbon emissions in the health sector. PloS One 2014; 9: e105040

[6] Hahm JS, Lee HL, Choi HS et al. Telemedicine system using a highspeed network: past, present, and future. Gut Liver 2009; 3: 247 - 251

[7] Kaltenbach T, Muto M, Soetikno R et al. Teleteaching endoscopy: the feasibility of real-time, uncompressed video transmission by using advanced-network technologies. Gastrointest Endosc 2009; 70: $1013-1017$

[8] Shimizu S, Itaba S, Yada S et al. Significance of telemedicine for video image transmission of endoscopic retrograde cholangiopancreatography and endoscopic ultrasonography procedures. J Hepato-biliarypancreatic Sci 2011; 18: $366-374$

[9] Chung SC, Li SS, Poon KO. Teleconferencing in endoscopic surgery. Hong Kong Medi J 1998; 4: 296 - 299

[10] Duty B, Okhunov Z, Friedlander J et al. Live surgical demonstrations: an old, but increasingly controversial practice. Urology 2012; 79 : 1185 e7-e11

[11] Shimizu S, Ohtsuka T, Takahata S et al. Remote transmission of live endoscopy over the Internet: Report from the 87th Congress of the Japan Gastroenterological Endoscopy Society. Digest Endosc 2016; 28: $0192-97$

[12] Cao MD, Shimizu S, Antoku Y et al. Emerging technologies for telemedicine. Korean J Radiol 2012; 13: (Suppl. 01): S21-30

[13] Kudo K, Shimizu S, Chiang T-C et al. Evaluation of Videoconferencing Systems for Remote Medical Education. Creative Education 2014; 5: $1064-1070$

[14] Go PM, Payne JHJr, Satava RM et al. Teleconferencing bridges two oceans and shrinks the surgical world. Surg Endosc 1996; 10: 105 106 


\section{Appendix 1: Feedback questionnaires (please answer the following questions)}

1. How was the image resolution?
a) Very good
b) Good
c) Poor
d) Very poor

2. How was the image movement?
a) Very smooth
b) Smooth
c) Slow (sluggish)
d) Very slow (sluggish)

3. How was the quality of sound?
a) Very good
b) Good
c) Poor
d) Very poor

4. How was the preparation?
a) Very easy
b) Easy
c) Time-consuming
d) Very time-consuming

5. How was the program?
a) Very good
b) Good
c) Poor
d) Very poor

6. Will you attend another teleconference session next time?
a) Yes
b) Probably yes
c) Probably no
d) $\mathrm{No}$

7. If you found any problem during the teleconference, please state here:

8. State your station:

9. State your occupation: medical doctor, engineer or other 